\title{
Condições afetivas e de relacionamento interpessoal em homens portadores de esquizofrenia em tratamento com haloperidol ou clozapina
}

\author{
Rejane Veiga Oliveira Johann \\ Universidade Luterana do Brasil \\ Cícero E. Vaz \\ Pontifícia Universidade Católica do Rio Grande do Sul
}

\begin{abstract}
RESUMO
Foi feito um estudo sobre condições afetivas e de relacionamento interpessoal de homens com diagnóstico prévio de esquizofrenia. Foram comparados os dados do Rorschach de dois grupos de participantes: Grupo 1, pacientes medicados com Haloperidol, $(n=14)$; Grupo 2, medicados com Clozapina (n=16). Os grupos constituíram-se de homens com recidiva da doença, internações prévias, em tratamento psiquiátrico ambulatorial no Hospital de Clínicas de Porto Alegre (HCPA). A faixa etária variou entre 18 e 59 anos. Os instrumentos utilizados foram: Operational Checklist for Psychotic Illness (OPCRIT) para definição do diagnóstico prévio de esquizofrenia e assim incluir na amostra apenas sujeitos com esta patologia, e a escolha da Técnica de Rorschach por ser um instrumento capaz de fornecer elementos para avaliação das condições afetivas e do relacionamento interpessoal. Foi feita a Análise de Variância (ANOVA) seguida do teste de Bonferroni, com nível de significância de $\mathrm{p} \leq 0.05$, sobre os dados do Rorschach. Os resultados do Rorschach permitem concluir que os pacientes medicados com Clozapina apresentam indicativos de controle geral sobre seus impulsos e instintos, capacidade de reparação, capacidades afetivas e emocionais para estabelecer relacionamentos interpessoais. Já os pacientes medicados com Haloperidol apresentaram apenas indicativos de controle geral sobre seus impulsos e instintos.
\end{abstract}

Palavras-chave: Rorschach; esquizofrenia; medicação antipsicótica.

\section{ABSTRACT \\ Affection and interpersonal relations on schizophrenic men medicated with haloperidol or clozapina}

An emotional condition and interpersonal relationships study has been made with men with schizophrenia previously diagnosed. A comparative study was prepared with Rorschach variables in two groups: Group 1 - patients medicated with Haloperidol $(n=14)$; Group 2 - patients medicated with Clozapina $(n=16)$. Groups were men who have either been previously diagnosed with schizophrenia, were recidivists, have been previously institutionalized or were under psychiatric treatment at the Hospital de Clínicas de Porto Alegre. The age of all subjects ranged from 18 to 59 years. The following tools were used in the study: The Operational Checklist for Psychotic Illness (OPCRIT) for schizophrenia previously diagnosed and include this way only patients with this pathology, the Rorschach Technique has been chosen, to be an instrument able to give elements to value emotional condition and interpersonal relationships. The Rorschach data was subject to a Variance Analysis (ANOVA) followed by a Bonferroni Test with a confidence interval of $\mathrm{p} \leq 0.05$. The results show that patients medicated with Clozapina present a better control over impulses and instincts, recovery capability, and affection and emotional capabilities for interpersonal relationships. Further more the patients medicated with Haloperidol present only better control over impulses and instincts.

Keywords: Rorschach; schizophrenia; antipsychotic medication.

\section{INTRODUÇÃO}

A esquizofrenia é, reconhecidamente, uma doença mental que causa um impacto devastador na vida das pessoas por ela acometidas e em seus familiares. Sua incidência anual é de 0,5 a 5,0 em cada dez mil pessoas (Abreu \& Gil, 2003; Manual Diagnóstico e Estatístico de Transtornos Mentais (DSM IV-TR) da American 
Psychiatric Association (APA), 2001/2002). Esta doença é definida pela presença de vários sintomas, no entanto, nenhum deles pode ser considerado patognomônico da doença. Para diagnosticar a esquizofrenia é necessário haver a presença de pelo menos dois sintomas psicóticos, por no mínimo um mês, e estes devem ficar proeminentes por pelo menos seis meses (Lara, Gama \& Abreu, 2004). Deverão ser descartados o transtorno esquizoafetivo e os transtornos do humor com aspectos psicóticos, bem como excluída a possibilidade de que a perturbação se deva ao uso de uma substância ou a uma condição médica geral (DSM-IVTR, 1997/2000; Classificação de Transtornos Mentais e de Comportamento (CID 10) da Organização Mundial de Saúde (OMS), 1992/1993).

O grupo de sintomas que compõe o espectro da esquizofrenia é dividido em três categorias: sintomas positivos (características ausentes em indivíduos saudáveis e presentes nestes pacientes), sintomas negativos (ausência de características que indivíduos saudáveis possuem) e sintomas desorganizados (características presentes tanto em indivíduos normais quanto em esquizofrênicos, porém nestes pacientes aparecem de forma mais caótica). São sintomas positivos de esquizofrenia as alucinações e delírios. Os sintomas negativos são embotamento afetivo, alogia, avolição e anedonia. Os sintomas desorganizados são comportamentos bizarros, afeto inapropriado e desorganização do pensamento (Lara e cols., 2004; Meltzer \& Fatemi, 2002; Razzouk \& Shirakawa, 2001).

Para o tratamento da esquizofrenia o uso de medicamentos antipsicóticos é indispensável. Esta medicação que ao longo de sua história já teve diversas denominações, tais como, tranqüilizantes maiores, neuroléptico, antipsicóticos típicos ou atípicos, hoje é denominada de antipsicótico de primeira ou de segunda geração (Bechelli, 2000; Fleischhacker, 2002/2005; Frota, 2001, Lara e cols., 2004; Nardi \& Bueno, 2000).

As pesquisas referentes à medicação antipsicótica de primeira ou de segunda geração têm o seu foco nas contribuições e contra-indicações destas medicações para a recuperação de pacientes esquizofrênicos (Bechelli, 2000; Fleischhacker, 2002/2005; Frota, 2001; Nardi \& Bueno, 2000; Louzã Neto, Shirakawa \& Barros, 1999; Shirakawa, Bressan \& Chaves, 2001; Stip, 2001). No entanto, a comunidade científica ainda carece de estudos dos efeitos destas medicações sob ponto de vista psicológico.

O tratamento com antipsicóticos reduz a sintomatologia em cerca de $70 \%$ dos pacientes. Os sintomas que mais respondem ao tratamento são os positivos, uma vez que os sintomas negativos são mais resistentes ao tratamento. Os antipsicóticos de segunda geração são eficazes na redução dos sintomas negativos da doença (Bechelli, 2000; Fleischhacker, 2002/2005; Lara e cols., 2004).

Dentre os antipsicóticos de primeira geração podese destacar o Haloperidol. Esta é ainda hoje uma das drogas mais utilizadas no tratamento de pacientes esquizofrênicos. Este medicamento foi sintetizado com o objetivo de atuar como um analgésico mais potente do que aqueles que existiam na época. A descoberta dos seus efeitos antipsicóticos ocorreu ao acaso (Bechelli, 2000).

A Clozapina, considerada um antipsicótico de segunda geração, foi a primeira droga que demonstrou superioridade em relação aos antipsicópticos de primeira geração, apresentando diferenças quantitativas e qualitativas. Sua aplicação traz vantagens não apenas ao tratamento de pacientes refratários ou sobre os sintomas negativos da doença. Ela mostra-se eficiente "em vários domínios psicossociais, incluindo a qualidade de vida e a prevenção de comportamentos suicidas" (Fleischhacker, 2002/2005, p. 73).

Como não existem antipsicóticos que não possuam efeitos indesejáveis, convém ser levado em consideração a "cautela, detecção precoce de efeitos adversos, diminuição de dose, troca por outra droga, suspensão de associações desnecessárias e modificação comportamental são estratégias recomendadas" (Abreu, Bolognesi \& Rocha, 2000, p. 43).

Este estudo se propõe a avaliar, sob o ponto de vista psicológico, o relacionamento afetivo e as relações interpessoais de pacientes portadores de esquizofrenia, associados ao tipo de medicamento antipsicótico em uso, Haloperidol ou Clozapina.

Consideraram-se as seguintes hipóteses alternativas:

H01: Pacientes esquizofrênicos que fazem uso de antipsicóticos de segunda geração apresentam melhores condições psicológicas do que aqueles que fazem uso de antipsicóticos de primeira geração quanto à capacidade para estabelecer relações interpessoais, diminuição do controle paranóide possibilitando a busca de contato humano e capacidade de reparação.

H02: Pacientes esquizofrênicos que fazem uso de antipsicóticos de segunda geração apresentam melhores condições psicológicas quanto ao controle geral de seus impulsos e instintos do que aqueles que fazem uso de antipsicóticos de primeira geração. 


\section{MÉTODO}

\section{Amostra}

A amostra foi constituída de dois grupos compostos por homens com diagnóstico prévio de esquizofrenia, com recidiva da doença, história de internações prévias, em tratamento psiquiátrico ambulatorial no Hospital de Clínicas de Porto Alegre. O Grupo 1 foi composto por 14 homens fazendo uso há, no mínimo, um ano e meio de Haloperidol, medicamento antipsicótico de primeira geração, e o Grupo 2 por 16 homens fazendo uso do antipsicótico de segunda geração, Clozapina, há no mínimo um ano e meio. A faixa etária situou-se entre 18 e 59 anos.

Foram adotados como critérios de exclusão: diagnóstico cruzado de esquizofrenia com doenças afetivas, pacientes que fazem uso concomitante de medicações de primeira e de segunda geração. Este último critério adotado limitou bastante o tamanho da amostra, uma vez que é muito comum o uso concomitante de medicamentos de primeira e de segunda geração por pacientes com esquizofrenia.

\section{Instrumentos}

Os pacientes foram avaliados por meio do Operational Checklist for Psychotic Illness (OPCRIT) para definição do diagnóstico de esquizofrenia, validado para o Brasil em trabalho conjunto da equipe do Hospital de Clínicas de Porto Alegre com a equipe de pesquisadores portugueses (Azevedo e cols., 1999). O OPCRIT é um instrumento que inclui 90 itens de sinais e sintomas presentes nas principais classificações psiquiátricas. Um programa de computador gera o diagnóstico de acordo com critérios estabelecidos por 12 sistemas de classificação nosológica (DSM IV, DSM III, DSM III-R, RDC, Feighner e cols. (St. Louis), Carpenter, Schneider, French, Taylor \& Abrams, Tsuang \& Winokur, Crow, Farmer, CID10). Ele é composto por itens que avaliam a resposta ao tratamento e dimensões de sintomas: 1) aparência e comportamento, 2) discurso e forma do pensamento, 3) afeto e aspectos associados, 4) crenças e idéias anormais, 5) percepções anormais (Wickham e cols., 2002). Ele possibilita a definição de diagnósticos aproximados para pesquisas de transtornos mentais severos (Williams, Farmer, Ackenheil, Kaufmann \& McGuffin, 1996). O OPCRIT é considerado um instrumento confiável, de rápida aplicação e de validade para pesquisas de doenças psiquiátricas (Craddock, Asherson, Owem, Williams \& Farmer, 1996).
Para a avaliação do controle geral dos impulsos, da capacidade de estabelecer relações interpessoais, das condições afetivas, da necessidade de a pessoa buscar contato humano, e da capacidade de reparação foi utilizada a Técnica de Rorschach. Esta técnica, de autoria de Hermann Rorschach, teve sua primeira edição completa e perfeita ao final de junho de 1921. Trata-se de uma técnica que possibilita fazer a avaliação dos elementos psicodinâmicos da pessoa de modo abrangente e global, uma vez que por meio dela se pode avaliar a estrutura da personalidade do indivíduo e o funcionamento de seus psicodinamismos, seus traços de personalidade, o funcionamento de suas condições intelectuais, o nível de ansiedade básica e situacional, a depressão, suas condições afetivas e emocionais, o controle geral, a capacidade para suportar frustrações e conflitos, integração humana, impulsos, instintos e reações emocionais (Vaz, 1997).

Foram levados em conta os seguintes dados quantitativos da Técnica de Rorschach:

a) A incidência de respostas de Forma pura $(\Sigma F)$ e de movimento animal (FM) para avaliação do controle geral e das reações impulsivas.

b) A incidência de respostas Cromáticas $(\mathrm{FC}, \mathrm{CF}$, C) e respostas de Movimento Humano $(\mathrm{M}+$, $\mathrm{M}-, \mathrm{M} \pm$ ) para avaliação da capacidade de estabelecer relações interperssoais.

c) A incidência de conteúdos percebidos em Sombreado de Textura $(\mathrm{Fc}, \mathrm{cF}, \mathrm{c})$ para avaliar as necessidades de a pessoa buscar contato humano.

d) A incidência das respostas de Cor acromática (FC', C'F, C') para avaliação da capacidade de reparação e de reações depressivas.

Foi realizada interpretação dinâmica dos resultados relacionando-os com os índices encontrados na literatura como esperáveis (normais) das variáveis quantitativas.

\section{Procedimentos}

O estudo foi realizado no ambulatório de Esquizofrenia do Hospital de Clínicas de Porto Alegre HCPA. A escolha da amostra dos pacientes foi feita após a confirmação do diagnóstico pela equipe médica por meio da aplicação do OPCRIT. Após a obtenção do Consentimento Livre e Esclarecido dos pacientes e de seu responsável (familiar), foram seguidos os seguintes procedimentos entrega de uma cópia do Consentimento Livre e Esclarecido ao paciente e a seu familiar, combinação de horários com os pacientes, aplicação individual da técnica de Rorschach. 


\section{RESULTADOS}

Foi feito estudo comparativo sobre os dados do Rorschach, entre os grupos: Grupo 1 composto de pacientes que fazem uso de medicamento antipsicótico de primeira geração - Haloperidol e Grupo 2 de pacientes que fazem uso de medicamento antipsicótico de segunda geração - Clozapina. Foi usada a Análise de Variância (ANOVA) seguida do teste de Bonferroni com nível de significância de $\mathrm{p} \leq 0,05$.

Realizadas as comparações múltiplas, foi confirmada no Rorschach a seguinte hipótese alternativa: Pacientes esquizofrênicos que fazem uso de antipsicóticos de segunda geração apresentam melhores condições psicológicas do que aqueles que fazem uso de antipsicóticos de primeira geração quanto à capacidade para estabelecer relações interpessoais, diminuição do controle paranóide possibilitando a busca de contato humano e capacidade de reparação.

Tanto o Grupo 1 como o Grupo 2 apresentaram número reduzido de respostas de movimento humano por protocolo (Grupo 1: $\Sigma \mathrm{M}=0.57$; Grupo 2: $\Sigma \mathrm{M}=$ 1,13). No entanto, o Grupo 1 (Haloperidol) teve um número muito reduzido destas respostas, denotando grandes dificuldades quanto à adaptação ao meio externo, bem como dificuldades em desenvolver adequado relacionamento interpessoal, uma vez que não demonstram capacidade de integração com as pessoas.

O Grupo 2 (Clozapina) revela adequado índice de respostas $\mathrm{M}+(\mathrm{M}+=88.50 \%)$, embora a diferença entre os grupos não seja estatisticamente significativa, clinicamente é um indicativo de que estas pessoas têm condições de estabelecer relacionamentos interpessoais com potencial empático. Neste caso, acredita-se que a medicação Clozapina tenha contribuído para isto.

A presença de respostas de movimento humano em um protocolo de Rorschach é interpretada como indicativo da expressão do mundo interno da pessoa, da capacidade criadora, do poder de adaptação ao meio externo, das condições de relacionamento humano. Um número muito reduzido de respostas de movimento humano evidencia estereotipia do pensamento. Sendo assim, um protocolo de Rorschach dentro da faixa clinica considerada normal, apresenta de 2 a 4 respostas de movimento humano. Entretanto, o tipo de movimento humano $(\mathrm{M}+, \mathrm{M} \pm, \mathrm{M}-)$ é que vai determinar a qualidade do relacionamento interpessoal que o sujeito possui recursos para estabelecer. Assim, pessoas que estabelecem relacionamentos interpessoais empáticos, com potencial para vinculação afetiva de- vem, em princípio, apresentar o índice mínimo de $80 \%$ de respostas de movimento humano de boa qualidade - M+ - (Vaz, 1997). Quanto mais baixo o índice de M num protocolo de Rorschach maiores dificuldades apresenta o paciente, como acontece com os pacientes do Grupo 1 (Haloperidol).

A cor cromática no Rorschach "tem o poder de mobilizar o mundo interno da pessoa, provocando-lhe reações afetivo-emocionais, com as mais variadas repercussões em todo o aparato psíquico da personalidade" (Vaz, 1997, p. 92). A capacidade de dar e receber afeto de forma adequada é verificada pela proporção $\mathrm{FC} \geq \mathrm{CF}+\mathrm{C}$. O Grupo 1 apresenta $\mathrm{FC}<\mathrm{CF}+\mathrm{C}$ $(0.36<0.57)$ indicando que, ao serem mobilizadas afetiva e emocionalmente, essas pessoas têm dificuldades para reagir de forma adequada e adaptada no relacionamento interpessoal, além das poucas condições de integração humana, já apontado no baixo índice de respostas de movimento humano.

$\mathrm{O}$ sombreado textura $(\mathrm{Fc}, \mathrm{cF}, \mathrm{c})$ é uma das variáveis do Rorschach interpretadas como necessidade de buscar contato humano, e em alguns casos, como carência afetiva, por parte do examinando. Os dois grupos apresentam respostas com esse determinante, demonstrando ser pessoas carentes de afeto, ou seja, sentem necessidade de carinho e apoio. Entretanto, trata-se de um afeto imaturo que mais busca receber do que dar afeto.

Os dois grupos apresentaram respostas de Cor Acromática (FC', C'F, C'). Porém, apesar de não existir diferença estatisticamente significativa entre os grupos, o Grupo 2 (Clozapina) se destaca por apresentar FC' > C'F + C' $(0.38>0.34)$, o que pode ser interpretado clinicamente como capacidade de reparação. Os integrantes do Grupo 1 (Haloperidol) apresentam FC' < C'F + C' $(0.14<0.51)$ denotando sinais de depressão. Vaz (1997) destaca que a presença de respostas de Cor Acromática em um protocolo de Rorschach indica que a pessoa tem tendência de evitar os estímulos que possam lhe mobilizar reações emocionais.

Não se confirmou a hipótese: Pacientes esquizofrênicos que fazem uso de antipsicóticos de segunda geração apresentam melhores condições psicológicas quanto ao controle geral de seus impulsos e instintos do que aqueles que fazem uso de antipsicóticos de primeira geração.

Não foram evidenciadas diferenças estatisticamente significavas entre os dois grupos quanto ao índice de respostas de forma pura $(\Sigma \mathrm{F})$ por protocolo 
de Rorschach. Todos apresentam adequado controle geral sobre seus impulsos e instintos ( $\Sigma \mathrm{F}$ entre $30 \mathrm{e}$ $50 \%$ ). O adequado controle geral sobre os impulsos e instintos de ambos os grupos pesquisados contraria a literatura que aponta um índice de forma pura $(\Sigma \mathrm{F})$ geralmente elevado (acima de 50\% do total das respostas) em protocolos de Rorschach de pessoas esquizofrênicas, sendo, na maioria dos casos, sinal de repressão (Vaz, 1997). No entanto, o baixo índice médio de respostas de movimento animal (FM) em ambos os grupos (Grupo $1=1.00$; Grupo $2=1.31$ ) indica que se trata de pessoas altamente defensivas e sem capacidade de iniciativa.

\section{CONCLUSÕES}

A partir dos resultados encontrados nesta pesquisa e com base em alguns aspectos da literatura, há dados que se destacam e que podem auxiliar no entendimento do paciente esquizofrênico em tratamento com Haloperidol e do paciente esquizofrênico em tratamento com Clozapina.

Ao analisar-se o funcionamento da personalidade de pacientes com diagnóstico de esquizofrenia, com base nos dados do Rorschach apontados pela literatura, constatam-se: inadequado controle geral sobre seus impulsos e instintos, dificuldades em estabelecer relacionamentos interpessoais, prejuízo quanto à capacidade de adaptação e integração com as pessoas. São pessoas que têm facilidade para perder o controle emocional e propensão a escapes agressivos. Denotam desejo de contato, mas não conseguem aproximação com as demais, devido ao controle paranóide; são pessoas imaturas, que mais buscam receber do que dar afeto às demais.

Os pacientes tratados com Haloperidol apresentam controle geral razoavelmente adequado sobre seus impulsos e instintos, contrariando, até certo ponto, a literatura no que tange à esquizofrenia, conforme achados no Rorschach. São pessoas com grandes dificuldades de adaptação ao meio externo, dificuldades em desenvolver adequado relacionamento interpessoal e não demonstram capacidade de integração humana, ratificando o que diz a literatura. Quando mobilizadas afetiva e emocionalmente têm dificuldades para reagir de forma adequada e adaptada em relacionamentos interpessoais. São pessoas que diante de uma situação de pressão e tensão externa podem perder o controle emocional com muita facilidade. Sentem necessidade de atenção, afeto e apoio.
Esses resultados permitem inferir que a medicação antipsicótica Haloperidol, do ponto de vista psicológico, está auxiliando apenas no controle geral sobre os impulsos e instintos deste grupo de pacientes estudado.

Já, os pacientes em tratamento há, no mínimo um ano e meio, com a medicação antipsicótica Clozapina apresentam, no Rorschach, controle geral sobre seus impulsos e instintos. Não denotam dificuldades quanto à adaptação ao meio externo, ou em desenvolver adequado relacionamento interpessoal, uma vez que demonstram razoável capacidade de integração com as pessoas. Apresentam relativas capacidades afetivas e emocionais para relacionamentos interpessoais. Revelam indicativos de carência afetiva, capacidade de reparação e não demonstram reações depressivas comprometedoras.

Pode-se deduzir que a medicação antipsicótica Clozapina pode estar auxiliando a esses pacientes no desenvolvimento de relacionamentos interpessoais de forma razoavelmente adequada e de reintegração social.

\section{REFERÊNCIAS}

Abreu, P. B., Bolognesi, G. \& Rocha, N. (2000). Prevenção e tratamento de efeitos adversos de antipsicóticos. Revista Brasileira de Psiquiatria, 22 (Supl. I), 41-44.

Abreu, P. \& Gil, A. (2003). Esquizofrenia. Em A. Cataldo Neto, G. J. C. Gauer \& N. R. Furtado (Orgs), Psiquiatria para estudantes de medicina (pp. 369-380). Porto Alegre: Edipucrs.

American Psychiatric Association. - APA. (2000). Diretrizes no tratamento da esquizofrenia. Porto Alegre: Editora Artes Médicas Sul.

American Psychiatric Association - APA. (2002). Manual Diagnóstico e Estatístico de Transtornos Mentais - DSM-IVTR (4 ${ }^{\mathrm{a}}$ ed. rev.). Porto Alegre: Editora ArteMed.

Azevedo, M. H., Soares, M. J., Coelho, I., Dourado, A., Valente, J., Macedo, A., Pato, M. \& Pato, C. (1999) Using consensus OPCRIT diagnoses: Na efficient procedure for best-estimate lifetime diagnoses. British Journal of Psichiatry, 175, 154-157.

Bechelli, L. P. C. (2000). Impacto e evolução do emprego dos neurolépticos no tratamento da esquizofrenia. Jornal Brasileiro de Psiquiatria, 49 (5), 131-147.

Craddock, M., Asherson, P., Owem, M. J., Williams, P. \& Farmer, A. F. (1996). Concurrent validity of the OPCRIT diagnostic system. Compariosn of OPCRIT diagnoses with consensus best-estimate lifetime diagnoses. Jornal Brasileiro de Psiquiatria, 169 (1). 58-63.

Fleischhacker, W. W. (2005). Tratamento farmacológico da esquizofrenia: uma revisão. Em M. Maj \& N. Sartorius. Esquizofrenia (2 ${ }^{\mathrm{a}}$ ed.) (pp. 71-101). Porto Alegre: ArtMed Editora.

Frota, L. H. (2001). Cinqüenta anos de medicamentos antipsicóticos em psiquiatria: I fenotiazinas alifáticas. Jornal Brasileiro de Psiquiatria, 50 (3-4), 121-141. 
Lara, D. R., Gama, C. S. \& Abreu, P. S. B. (2004). Esquizofrenia. Em F. Kapczinski, J. Quevedo \& I. Izquerdo e col. Bases biológicas dos transtornos psiquiátricos ( $2^{\mathrm{a}} \mathrm{ed}$.) (pp. 285-307). Porto Alegre: Editora Artmed.

Louzã Neto, M. R., Shirakawa, I. \& Barros, L. (1999). Esquizofrenia: dois enfoques complementares. São Paulo: Lemos Editorial.

Meltzer, H. Y. \& Fatemi, S. H. (2002). Esquizofrenia. Em M. H. Ebert, P. T. Loosen \& B. Nurcombe. Psiquiatria - diagnóstico e tratamento (pp. 259-275). Porto Alegre: Editora Artmed.

Nardi, A. E. \& Bueno, J. R. (2000). Esquizofrenia: avanços no tratamento. Em J. R. Bueno \& A. E. Nardi (Orgs.), Diagnóstico e Tratamento em Psiquiatria (pp.171-218). Rio de Janeiro: Editora Medsi.

Organização Mundial de Saúde - OMS. (1993). Classificação de transtornos mentais e de comportamento - CID-10. Porto Alegre: Editora Artes Médicas.

Razzouk, D. \& Shirakawa, I. (2001). A evolução dos critérios diagnósticos da esquizofrenia. Em I. Shirakawa, A. C. Chaves \& J. Mari (Orgs.), O desafio da esquizofrenia (pp. 15-23). São Paulo: Lemos Editorial.

Shirakawa, I., Bressan R. A. \& Chaves A. C. (2001). Tratamento farmacológico da esquizofrenia. Em I. Shirakawa, A. C. Chaves
\& J. Mari. O desafio da esquizofrenia (pp. 135-148). São Paulo: Lemos Editorial.

Stip, E. (2001). Antipsicóticos nuevos: temas y controversias. Tipicidad de los antipsicóticos atípicos. Revista de Psiquiatria del Uruguay, 65 (1).70-91.

Vaz, C. E. (1997). O Rorschach - Teoria e desempenho ( $3^{\mathrm{a}}$ ed.). São Paulo: Editora Manole.

Vaz, C. E. (2002). Z-Teste: Técnica coletiva de Zulliger: Forma coletiva ( $2^{\mathrm{a}}$ ed.). São Paulo: Editora Casa do Psicólogo.

Wickham, H., Walsh, C., Asherson, P., Gill, M., Owen, M. J., McGuffin, P., Murray, R. \& Sham, P. (2002). Familiaty of clinical characteristics in schizophrenia. Journal of Psychiatric Research, 36, 325-329.

Williams, J., Farmer, A. E., Ackenheil, M., Kaufmann, C. A. \& McGuffin, P. (1996). A multicentre inter-rater reliability study using the OPCRIT computerized diagnostic system. Psychological Medicine, 26 (4). 775-783.

Recebido: $15 / 04 / 2006$ Revisado: 07/07/2006 Aceito: $17 / 07 / 2006$

\section{Nota:}

${ }^{1}$ Artigo resultante da Tese de Doutorado "Características de personalidade de homens portadores de esquizofrenia em tratamento com Haloperidol ou Clozapina”. Apoio institucional CAPES.

\section{Sobre os autores:}

Rejane Veiga Oliveira Johann: Psicóloga, Doutora em Psicologia pela PUCRS, Professora do curso de graduação em Psicologia da Universidade Luterana do Brasil (ULBRA), Gravataí. Endereço eletrônico: rejanejohann@ terra.com.br.

Cícero E. Vaz: Psicólogo, Doutor em Psicologia, Professor Titular do Programa de Pós-Graduação da Faculdade da Pontifícia Universidade Católica do Rio Grande do Sul (PUCRS), Porto Alegre; Pesquisador - Produtividade em Pesquisa do CNPq. 